\title{
Hábitos alimentarios y estado nutricional de los estudiantes de las universidades de la Región Lima - 2015
}

\author{
Dietary habits and nutritional status of students in the universities of the \\ Lima Region - 2015 \\ Elsa Carmen Oscuvilca Tapia ${ }^{1, a}$, Walter Jesús Sosa Hijar, ${ }^{1, b}$, Olga Cáceres Estrada, ${ }^{1, c}$, \\ William Rogelio Peña Ayudante ${ }^{1, d}$, Jacinto Jesús Palacios Solano ${ }^{1, e}$
}

\section{RESUMEN}

Objetivo: Determinar la relación entre los hábitos alimentarios y estado nutricional de los estudiantes de las universidades de la Región Lima - 2015. Materiales y Métodos: Estudio de enfoque cuantitativo cualitativo tipo correlacional descriptivo, transversal, retrospectivo realizado en una muestra de 360 estudiantes de las universidades de la Región Lima; cuenta con el soporte de las teorías de Cloningery Bandura; los instrumentos para la recolecta de datos válidos y confiables mediante el juicio de expertos y prueba piloto respectivamente. Para el análisis de los datos se empleó la prueba Chi cuadrado con el programa SPSS versión 17.0. Resultados: El $46.1 \%$ tiene hábitos alimentarios poco adecuados, el $28,6 \%$ hábitos adecuados y el $25,3 \%$ hábitos inadecuados. El estado nutricional de los universitarios reporta el IMC de $57.8 \%$ normal, el $21,4 \%$ delgadez y el $20,8 \%$ obesidad. Conclusión: Los hábitos alimentarios poco saludables pueden ser los responsables de los problemas nutricionales que presentan los estudiantes como la delgadez y obesidad. La relación entre los hábitos alimentarios y estado nutricional de los estudiantes universitarios, genera una linea de investigación en el área de nutrición y salud mental, en procura de fomentar la cultura de la alimentación saludable.

Palabras clave: Hábitos alimentarios, estado nutricional, universidad saludable.

\begin{abstract}
Objective: To determine the relationship between dietary habits and nutritional status of the students of the Lima - 2015 Region. Materials and Methods: A quantitative qualitative approach, descriptive, cross - sectional, retrospective, correlational study carried out on a sample of 360 students from the Universities of the Lima Region; Counts on the support of the theories of Cloningery Bandura; The instruments for the collection of valid and reliable data through expert judgment and pilot testing respectively. For the analysis of the data the Chi square test was used with the program SPSS version 17.0. Results: $46.1 \%$ had poor eating habits, $28.6 \%$ had adequate habits and $25.3 \%$ had inadequate habits. The nutritional status of university students reports a BMI of $57.8 \%$ normal, $21.4 \%$ of leanness and $20.8 \%$ of obesity. Conclusion: Unhealthy eating habits may be responsible for the nutritional problems presented by students such as thinness and obesity. The relationship between eating habits and nutritional status of university students generates a line of research in the area of nutrition and mental health, in order to promote a healthy eating culture.
\end{abstract}

Key words: Eating habits, nutritional status, healthy university.

'Facultad de Medicina Humana, Universidad Nacional José Faustino Sánchez Carrión, Huacho, Perú. ${ }^{a}$ Docente Principal, Docente en Salud Pública,

${ }^{b}$ Docente Asociado. Licenciado en Bromatología y Nutrición, ${ }^{\circ}$ Docente Principal Mstra. Investigación y Docencia Universitaria, ${ }^{\circ}$ Docente Asociado. Maestro en Salud Reproductiva,

${ }^{\circ}$ Docente Principal. Magíster en Fisiología. 


\section{INTRODUCCIÓN}

El inicio del periodo universitario suele coincidir con el final de la adolescencia y el paso a la edad adulta, por ello, aun siendo muchos de los estudiantes ya adultos, se suscita en ellos los cambios fisiológico y psicológicos característicos de la adolescencia tardía. Fundamentalmente, los universitarios más jóvenes, que están todavía en proceso anabólico de crecimiento y de reposición de nutriente en las estructuras corporales, y con unos requerimientos mayores que en el resto de las etapas de la vida, pueden ver condicionado su estado nutricional por cualquier alteración en el patrón de dieta y/o estilo de vida. Travé, T. (2013), Cossio, M. et al. (2011)

A los cambios propios de la adolescencia, se mantienen en el adulto joven que modifican sus hábitos alimentarios, debido al acceso a la vida universitaria y el alejamiento en muchas ocasiones del entorno familiar, la adquisición de nuevas responsabilidades en la compra de los alimentos, la elaboración de sus menús, la organización de unos horarios de comidas que resultan ser bastante irregulares, los efectos de la "globalización" que son aún más acusados en la población joven y el mayor numero de comidas que se realizan en el comedor institucional y de ocio. Musitu, G. \& García, F. (2001). En la población universitaria se suele asociar su patrón alimentario y de estilo de vida con la irregularidad en el patrón de comidas, el consumo elevado de comida "rápida" y de bebidas con contenido alcohólico, el seguimiento de dietas nutricionalmente inadecuadas con baja densidad nutricional, la elevada incidencia de los transtornos de la conducta alimentaria, etc. que, unido a un incremento de los requerimientos nutricionales, dificultarán el seguimiento de dietas saludables y equilibradas.

Por otro lado, se producen unos estilos de vida muy diferenciados de unos y otros, que van desde el sedentarismo hasta la práctica del deporte de competencia (Dalmau 2012), (Burriel 2013) dificultando aún más la valoración nutricional de este grupo y los mensajes correctos a transmitir. Los adolescentes europeos, especialmente los españoles consumen la Dieta Mediterránea (DM) convertido en un modelo de alimentación y estilo de vida saludable que se relaciona con una mayor esperanza de vida, asociada con un efecto protector frente a diferentes enfermedades crónicas. Representa un estilo de vida que engloba, además, de la combinación de alimentos, elementos culturales y de estilo de vida del proceso de selección, procesamiento y consumo, como son la priorización de los alimentos frescos, locales y estacionales; de las actividades culinarias de la socialización en las comidas, de la actividad fisica regular, del descanso en forma de siesta y toda una manera de vivir que forma parte de la herencia cultural de los paises mediterráneos; sin embargo en estudios realizados en los estudiantes universitarios de Alicante y Navarra se ha visto que los alumnos presentan una baja adherencia a la dieta mediterránea. (Trichopoulou 2003), OMS (2004).

La problemática actual acerca de los desórdenes en la calidad de alimentación y las prácticas de rutinas de ejercicios/sedentarismo, provocan consecuencias tales como la desnutrición, sobrepeso y obesidad, riesgosas para salud y el desarrollo en la niñez y adolescencia. Ledo-Varela M (2011) y la Organización Mundial de la Salud, consideran la obesidad como uno de los problemas de salud pública más importante en el mundo por las graves consecuencias para la salud a corto y largo plazo.

El sobrepeso y la obesidad adolescente aumentan el riesgo de padecer obesidad en la edad adulta, así como la prevalencia de factores de riesgo cardiovascular clásicos, como el perfil lipídico aterogénico, la hipertensión arterial, la intolerancia a la glucosa y la diabetes tipo 2 . Se ha demostrado una agregación de los diversos factores de riesgo cardiovascular que actúan sinérgicamente. Por otro ello, el problema del sobrepeso y la obesidad trasciende el ámbito científico, siendo con frecuencia tratado en los medios de comunicación. Las modificaciones cuantitativas y cualitativas de las pautas de alimentación tradicional, asociadas a hábitos que conducen a una reducción de la actividad fisica, son las principales responsables de esta situación.

En consecuencia, las enfermedades crónicas no trasmisibles relacionadas a la alimentación y nutrición representan uno de los mayores problemas de salud pública en el mundo. Así, en el año 2001 fue la principal causa de aproximadamente $60 \%$ de las muertes en todo el mundo. Aproximadamente la mitad de ellas, se atribuyeron a enfermedades cardiovasculares. La obesidad y la diabetes también mostraron tendencias preocupantes no solo por efectar a una amplia proporción de la población, sino tambien, por su aparición en estadios cada vez más tempranos del ciclo de vida.

Las enfermedades crónicas no trasmisibles y su respectiva asociación con los estilos de vida, entre ellos los factores ligados a los hábitos y consumos que implican patrones de comportamientos propios de un grupo social, en tiempo y espacio definido; está adquiriendo un agran importancia en paises en desarrollo, a medida que se toma más conciencia del aumento de la frecuencia de las diversas enfermedades crónicas no transmisibles.

La Organización Mundial de la Salud, menciona en sus diversas publicaciones, que contrariamente el pensamiento general, las enfermedades crónicas no transmisibles vienen afectando de manera creciente a los paises en vías de desarrollo. Así, el $79 \%$ de las muertes producidas mundialmente y atribuidas a las 
enfermedades crónicas no transmisibles se produjeron en paises en vias de desarrollo. Uno de los factores de riesgo que más se relaciona con las enfermedades crónicas no trasmisibles es el sobrepeso y la obesidad. En América, la obesidad merece especial atención, ya que es en sí misma una enfermedad crónica y a la vez un reconocido factor de riesgo de muchas otras enfermedades. La epidemia de obesidad en nuestro continente se ha asociado con el crecimiento económico, la rápida urbanización y los subsecuentes cambios en los estilos de vida, entre ellos, el incremento en el consumo de grasa, azúcar, granos refinados y la reducción de la actividad física diaria.

En este mismo contexto, siendo la obesidad y el sobrepeso condiciones de salud que han presentado un aumento progresivo en las últimas tres décadas, tanto en países desarrollados como en los países en desarrollo; se denominan dicho fenómeno como "epidemia".

Similarmente, el Instituto Nacional de Salud. (2006) mediante el Centro Nacional de alimentación y Nutrición reportó datos preocupantes de prevalencia de sobrepeso en mujeres en edad fértil, constituyéndose en más de la tercera parte de este grupo etario; siendo los departamento más afectados Tacna (40\%), Lima $(38,8 \%$ ) y Arequipa $(37,3 \%)$ por la ingesta excesiva de carbohidratos acompañada de un mayor sedentarismo.

Al respecto, en el territorio peruano, muestra que la práctica de actividad deportiva regular en las zonas urbanas es escasas; siendo los más afectados los menores de 30 años, mujeres y los residentes del área metropolitana de la capital limeña (Seclén\&Jacoby, 2003). En materia de nutrición y alimentación, se ha detectado como problema principal de los adultos el mantener hábitos alimenticios y nutricionales poco saludables. Sin embargo, no se conocen con exactitud la magnitud de la población universitaria que padece mal nutrición por esceso o por déficit. Por estas razones el objetivo de la investigación es determinar la relación entre los hábitos alimentarios y estado nutricional de los estudiantes de las universidades de la Región Lima 2015. La presente investigación se justifica por la relevancia social, que obliga el abordaje transdisciplinaria ante la necesidad de desarrollar un marco establecido por la Organización Mundial de la Salud, la investigación dirigida al grupo vulnerable logrará predecir la calidad de vida durante años subsecuentes, con el propósito de disminuir las tasas de morbi mortalidad, el soporte teórico de la Teoría Tridimensional de Personalidad de Cloninger (1987) y teoría cognitiva social de Bandura, (198\&) contribuyen a buscar nuevas líneas de investigación en el área nutricional de los universitarios.

\section{MATERIALES Y MÉTODOS:}

Tipo de investigación. Según el problema y los objetivos, la investigación fue de enfoque cuantitativo cualitativo, tipo descriptivo correlacional. De acuerdo al tiempo de ocurrencia de los hechos y registro de la información fue retrospectiva y transversal.

Área de estudio: Se desarrolló en las universidades públicas de la Región Lima.

Población : :7200 distribuidos de la siguiente manera: Universidad Nacional José Faustino Sánchez Carrión: 6850 y Universidad Nacional de Barranca: 350 .

Tamaño de muestra: 360 estudiantes universitarios determinados con la fórmula para población finita y nivel de confianza deseado.

\section{DETERMINACIÓN DE LA MUESTRA: POBLACIÓN FINITA Y NIVEL DE CONFIANZA DESEADO}

\begin{tabular}{|c|c|}
\hline $\mathrm{N}$ & 7200 \\
\hline $\mathrm{Z}$ & 1.960 \\
\hline $\mathrm{p}$ & 0.5 \\
\hline $\mathrm{q}$ & 0.5 \\
\hline $\mathrm{E}$ & 0.05 \\
\hline $1-\mathrm{\alpha}$ & $95 \%$ \\
\hline $\begin{array}{l}\text { Error } \\
\text { Muestral }\end{array}$ & $5 \%$ \\
\hline
\end{tabular}

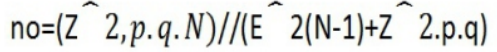

Tamaño de muestra: 360

Tipo de muestreo: Aleatorio simple.

Criterios de inclusión

- universitarios matriculados en el semestre académico 2015 - II.

- universitarios que accedan a participar en la investigación.

\section{Criterios de exclusión}

- universitarios con asistencia libre o no regular.

- universitarios que no aceptan participar en la investigación.

Técnicas e instrumentos de recolección de datos

La información se obtendrá a través del uso de técnicas cuantitativas, aplicados a los estudiantes universitarios, a fin de obtener de manera integral la información sobre los hábitos alimentarios respecto a la frecuencia de consumo de alimentos (frutas, verduras, legumbres y hortalizas, carnes y pescados).

Para recolectar la información del estado nutricional se consideran los datos antropométricos (peso y 
talla) siguiendo las normas de la Organización Mundial de Salud. El peso/talla se efectuó con el vestido y sin zapatos con una balanza Tanita TBF 300GS.

El indice de masa corporal (IMC) se calculó a partir de

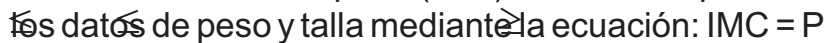
$(\mathrm{kg}) / \mathrm{T} 2(\mathrm{~m})$ se clasifica en 3 categorías: peso insuficiente (delgadez). IMC<18,5; normopeso, (18,5

IMC 24,9) y obesidad (IMC 30). El diámetro de cintura y cadera se efectuó con una cinta métrica de material inextensible (intervalo $0-150 \mathrm{~cm}$ ).

Validez del instrumento: Se determinó mediante el juicio de expertos en el área de Nutrición, Psicología, Sociología y Medicina Humana, con el coeficiente $R$ de Finncuyo acuerdo fortísimo de 0.92

Los procedimientos para la recolección de información, fueron autorizados por las autoridades y por el sujeto de investigación, a través del consentimiento informado.

\section{Técnicas, análisis y procedimiento de datos}

La información codificada mediante la construcción de un libro de códigos, fue ingresada a Excel y exportada al software estadístico SPSS versión 17.0 (StacticalPakageforthe Social Sciencies) para Windows XP; la estadística descriptiva identificó hábitos alimentarios y estado nutricional. La información se presentó en cuadros de una o doble entrada con sus respectivos gráficos. Los puntos de corte determinados para los hábitos alimentarios, según la escala de Estaninos fueron 29 - 94; Inadecuado; 95 - 101: poco adecuado y de 102 - 145: adecuado, aplicando la fórmula:

\section{$X \quad 0.75^{*} \mathrm{DS}$}

Donde:

\section{X : Media Aritmética}

\section{DS : Desviación Estándar}

\section{RESULTADO:}

\section{Características de la muestra.}

Se incluyeron en el estudio 360 universitarios, de los cuales el $47,5 \%$ son mujeres y el $52,5 \%$ varones. La media de edad fue de 22 años, concentrados mayoritariamente en el VI y VII ciclo, tienen el estado civil soltero el $88.3 \%$ y casado el $11,7 \%$ de las carreras de Medicina Humana, Enfermería, Derecho, Economía, Contabilidad, Ingeniería de Sistemas, Trabajo Social, Educación y Obstetricia. De ellos el $46.1 \%$ tiene hábitos alimentarios poco adecuados, el $28.6 \%$ hábitos alimentarios adecuados y el $25.3 \%$ hábitos alimentarios inadecuados.
El estado nutricional de los universitarios reporta el Indice de masa corporal (IMC) de 57,8\% normal, el $21,4 \%$ delgadez y el $20.8 \%$ obesidad.

La mayoría de los universitarios reporta el Indice de masa corporal (IMC) de $57.8 \%$ normal, el $21.4 \%$ delgadez y el $20.8 \%$ obesidad.

La mayoría de los estudiantes universitarios tienen los hábitos alimentarios poco adecuados en las dimensiones: alimentación consumida, alimentación por bienestar psicológico y calidad de la alimentación.

TABLA 1: Dimensiones de los hábitos alimentarios.

\begin{tabular}{|c|c|c|c|c|c|c|c|}
\hline \multirow[b]{2}{*}{ DIMENSIONES } & \multicolumn{7}{|c|}{ HÁBITOS ALIMENTARIOS } \\
\hline & $\operatorname{inade}_{n}$ & $\underset{\%}{\text { cuado }}$ & $\underset{n}{\operatorname{poco}}$ & $\underset{\%}{\operatorname{adec} u a d o}$ & $\underset{n}{\operatorname{adec}}$ & $\underset{\%}{\text { cuado }}$ & $\underset{\text { n }}{\text { TOTAL }}$ \\
\hline Alimentación consumida & 74 & 20.6 & 154 & 42.8 & 132 & 36.6 & 360100.0 \\
\hline Por bienestar psicológico & 70 & 19.4 & 168 & 46.7 & 122 & 33.9 & 360100.0 \\
\hline Calidad de la alimentación & 86 & 23.9 & 150 & 41.7 & 124 & 34.4 & 360100.0 \\
\hline
\end{tabular}

Fuente: Estudiantes de las universidades de la Región Lima - 2015

En la dimensión alimentación consumida, el 53.89\% refieren que pocas veces cocinan con mucho aceite, el $52.22 \%$ no consumen alimentos precocinados en vez de alimentos frescos, el $52.78 \%$ no consume carne de cerdo, el $37.50 \%$ consume ensaladas, el $47.22 \%$ consume cereales, el $42.22 \%$ no consume frutas en latas, el $57.78 \%$ no consumen golosinas, el $37.78 \%$ consumen gaseosas, el $57.78 \%$ no consumen frituras (salchipollos y salchipapas) y el $24.17 \%$ consumen hamburguesas.

En la dimensión alimentación por bienestar psicológico el $37.50 \%$ tienen en cuenta al restaurante que expeden comida que engorda, el 38.89\% está pendiente de los alimentos que engordan, el $36.39 \%$ verifica en las etiquetas de los alimentos la cantidad de calorías, el $66.67 \%$ controla a diario el consumo en exceso, el $29.17 \%$ consume alimentos cuando esta triste, el $30.28 \%$ consume alimentos cuando tienen ansiedad, el $22.78 \%$ siente hambre cuando esta aburrido/a.

En la dimensión conocimiento sobre la calidad de su alimentación, el $14.44 \%$ no ingieren 8 vasos de agua al día, el $36.94 \%$ pocas veces ingieren lácteos, quinua, el $54.17 \%$ muchas veces ingieren avena, el $36.39 \%$ consumen tallarines, el $38.89 \%$ pocas veces consumen pescados, el $38.33 \%$ carne de pollo y el $36.94 \%$ consumen frutas.

TABLA 2: Contingencia entre los hábitos alimentarios y el IMC.

\begin{tabular}{|c|c|c|c|c|c|}
\hline \multirow{2}{*}{\multicolumn{2}{|c|}{ Hábitos alimentarios }} & \multicolumn{3}{|c|}{ Índice de Masa Corporal } & \multirow[b]{2}{*}{ Total } \\
\hline & & Delgadez & Normal & Obesidad & \\
\hline Inadecuado & n & 17 & 54 & 20 & 91 \\
\hline \multirow[t]{2}{*}{ Poco Adecuado } & $\mathrm{n}$ & 24 & 102 & 40 & 166 \\
\hline & $\%$ & $6.7 \%$ & $28.3 \%$ & $11.1 \%$ & $46.1 \%$ \\
\hline \multirow{2}{*}{ Adecuado } & $\mathrm{n}$ & 36 & 52 & 15 & 103 \\
\hline & $\%$ & $100.0 \%$ & $14.4 \%$ & $4.2 \%$ & $28.9 \%$ \\
\hline$\overline{\text { Total }}$ & $\begin{array}{l}\mathrm{n} \\
\%\end{array}$ & $\begin{array}{c}77 \\
21.4 \%\end{array}$ & $\begin{array}{c}208 \\
57.8 \%\end{array}$ & $\begin{array}{c}75 \\
20.8 \%\end{array}$ & $\begin{array}{c}360 \\
100.0 \%\end{array}$ \\
\hline
\end{tabular}

Julio - Setiembre 201635 
La relación entre los hábitos alimentario y estado nutricional de los estudiantes de las universidades de la Región Lima resultó significativa debido a que la prueba no paramétrica Chi cuadrado $=17.121, \mathrm{gl}=4$, $p=0.002$

Las frecuencias observadas se sometieron a la siguiente prueba de hipótesis:

Hipótesis:

$\mathrm{H}_{\mathrm{o}}$ : No existe relación entre los hábitos alimentarios y el IMC de los estudiantes de la Región Lima.

$\mathbf{H}_{1}$ : Existe relación entre lo hábitos alimentarios y el IMC de los estudiantes de la Región Lima.

Nivel de significancia: Error tipo I, $\alpha=0.05(5 \%)$. Esto es, el error que se cometería al rechazar la $\mathrm{H}_{\mathrm{o}}$, siendo esta verdadera.

Prueba Estadística: Prueba no parametrica Chi cuadrado.

Decisión Estadística: El Chi cuadrado observado es $\mathrm{Ji}^{2}=17.121$ con $\mathrm{GL}=4$, al cual le está asociado un valor de probabilidad, $P=0.002$. Puesto que este valor $P$ es menor o igual que $\alpha$, se concluye en rechazar la $H_{\circ}$. Es decir existe relación entre los hábitos alimentarios y el IMC de los estudiantes de la Región Lima.

\section{DISCUSIÓN:}

Siendo el índice de masa corporal que indica el estado nutricional de la persona considerando dos factores elementales: su peso actual y su altura, se acepta internacionalmente, como criterio diagnóstico, con elevada especificidad y sensibilidad variable para la detección de sobrepeso, obesidad, peso abajo. Ha transcurrido el tiempo, la obesidad, la delgadez en los universitarios ya no son exclusivos de los países ricos, paradójicamente, es un problema de salud pública. En la presente investigación la obesidad y la delgadez relacionado con los hábitos alimentarios poco adecuados de los universitarios de la Región Lima, se debe al consumo de alimentos durante el receso de papa rellena, hamburguesas, galletas, aperitivos, dulces y salados aunque también consumen yogurt, jugos de fruta, además reconocen que tienen una alimentación inadecuada debido el tiempo insuficiente, para almorzar y cenar y por la poca disponibilidad de comedores universitarios. Por el horario de teoría o práctica muchos estudiantes no desayunan y se duermen en clases, otro grupo no almuerzan a la hora, por los horarios académicos dependientes de disponibilidad de aulas, es decir en las universidades de la Región Lima aun, no entra en vigencia los determinantes de la salud establecidos por la Organización Mundial de la Salud y adaptados por el Ministerio de Salud, respecto a las necesidades prioritarias de promoción de la salud, la alimentación y nutrición saludable en la comunidad universitaria que deben involucrar acciones conducentes a mejorar los hábitos alimentarios, como parte de un estilo de vida saludable; así mismo la modulación del expendio de productos alimentarios en el campus universitario, concordante con el derecho a la alimentación en el marco de los Acuerdos Internacionales suscritos para alcanzar la seguridad alimentaria.

En relacióna los datos sobre de los hábitos alimentarios, son semejantes en los alumnos de todas las carreras, lo que demuestra que el conocimiento de nutrición no implica necesariamente cambios hacia una dieta y estilos de vida más saludables. (Úbeda Martin N. 2006), y cada vez los hábitos nutricionales son bastantes deficitarios, llamando la atención especialmente la escasez de consumo de frutas y verduras, el bajo porcentaje de estudiantes que desayuna y la mala calidad del mismo, la escasez de consumo de aceite de oliva, pescado, lácteos y legumbres y por supuesto, la alarmante falta de ejercicio físico (Torres Manrique Blanca, Dura Ros María Jesús 2011). Los estudiantes de la Universidad Católica de la Santísima Concepción identifican la importancia que presenta la alimentación en su etapa académica, sin embargo, se percibe que la falta en al disponibilidad de tiempo es uno de los mayores condicionantes en la calidad de la alimentación recibida. El plantel no es percibido en forma favorable para mantener una alimentación saludable, esto se debería a la distribución horaria de la carga de la carga académica (Troncoso (2011))

En relación al IMC, los datos sobre el sobrepeso y obesidad, es mayor en los adultos jóvenes (62,3\%), los determinantes sociales del esceso de peso según el grupo de edad fueron: no ser pobre, vivir en el área y ser mujer. (Álvarez y Sánchez. 2012), y que el número de ingestas alimentarias diarias y el consumo de macronutrientes determinaron que el $50 \%$ presentó sobrepeso y un $40 \%$ grasa corporal elevada. (Pi y Vidal 2015). Similar situación en Colombia 42 estudiantes $(27,3 \%)$ están con sobrepeso y $7,8 \%$ con obesidad mórbida. (Laguado y Gómez 2014), similar al estudio de (Nuñez y Carranza 2012), en el que 76 de los 126 estudiantes analizados (60\%) tenían IMC entre 18,5 y $24,9 \mathrm{~kg} / \mathrm{m}$ (normal) y el $11 \%$ se encontraron en límites de 25 a $26,9 \mathrm{~kg} / \mathrm{m} 2$, por lo que se deduce que tienen sobrepeso. El estudio de Coral e Itas (2010) sostiene que, el estado nutricional y hábitos alimentarios de estudiantes de la Escuela de Enfermería de la Facultad Ciencias de la Salud tuvieron una prevalencia de desnutrición del $2,8 \%$, el sobrepeso alcanzó al $20,6 \%$ y la obesidad al $3,3 \%$.

No hay similitud con la evaluación nutricional en estudiantes de Enfermería, quienes el $80 \%$ de las participantes presentan un IMC dentro de los valores normales $(21,3 \mathrm{~kg} / \mathrm{m} 2)$ mientras que el $11,1 \%$ presentaban bajo peso, el 8,9\% sobrepeso y ninguna 
presentaba obesidad (Iglesias y Escudero 2010), la diesta de los estudiantes universitarios es ligeramente hipocalórica, más del $91 \%$ de los estudiantes se encuentra en una situación de "necesidad de cambios en la dieta" hacia patrones más saludables (Cervera y Serrano (2013).

No es similar la relación significativa del presente estudio con el hallazgo en los estudiantes de la UNMSM de Perú, debido a que no es significativa $(p=$ 0.185). (Ferro y Maguiña 2012).

Los hábitos alimentarios en los estudiantes universitarios, según Teoría Tridimensional de Personalidad de Cloningerse basan en tres dimensiones de la personalidad, cada una de las cuales estaría definida según un neurotransmisor especifico presente en las vías neuronales del sistema cerebral. Estas dimensiones de personalidad se pueden presentar en diferentes combinaciones en los seres humanos y estar genéticamente determinantes dando cuenta, por lo tanto, de la organización funcional que subyace a la personalidad de cada individuo. Dichas dimensiones son: la búsqueda de novedad, la evitación del daño y dependencia de la recompensa, complementándose con la teoría cognitiva social de Bandura, por la interacción recíproca entre las influencias ambientales externas, la conducta y los factores personales y cognitivos, donde el concepto de "autoeficacia" o percepciones que tiene el individuo de sobre su capacidad de actuar, adquiere un papel central elemento explicativo de la adquisición, mantenimiento y cambio de la conducta.

\section{CONCLUSIÓN}

Los hábitos alimentarios poco saludables pueden ser los responsables de los problemas nutricionales que presentan los estudiantes como la delgadez y obesidad, a esto se suma la falta de actividad física que por falta de tiempo o por los horarios poco flexibles que tienen los estudiantes.

La relación significativa entre los hábitos alimentarios y estado nutricional de los estudiantes universitarios, genera una línea de investigación en el área de nutrición y salud mental, en procura de fomentar la cultura de la alimentación saludable.

Conflicto de interés: Los autores declaran no tener conflictos de interés de ninguna naturaleza.

\section{AGRADECIMIENTO}

Los investigadores agradecen por su colaboración al Dr. Armando Vásquez Aristizabal por su colaboración en aplicación del instrumento de recolecta de datos. 


\section{REFERENCIA BIBLIOGRÁFICA}

Álvarez D. , Sánchez J., Gómez G., Tarqui C. , (2009 2010) Sobrepeso y obesidad: prevalencia y determinantes sociales dele sceso de peso en la población peruana. RevPeruMedExp Salud Publica. 2012; 29(3):303 - 13.

Bandura, A. (1986). Fundamentos sociales de pensamiento y acción. A sociales la teoría cognitiva. EnglewoodCliffs, NJ: Prentice Salas (trad. elenco. Barcelona, Martinez Rosa, 1987).

Bennasar M. (2012). Estilos de vida y salud en estudiantes universitarios: la universidad como entorno promotor de la salud. Universitat de les Illes Balears. España.

Cervera F. Faustino, Serrano R. Ramón (2013). Hábitos alimentarios y evalaución nutricional en una población Universitaria, Facultad de Enfermería. Universidad de Catilla- La Mancha. Albacete. España.

Coral P., Itas M., (2010) Estado nutricional y hábitos alimentarios de estudiantes de la Escuela de Enfermería de la Facultad Ciencias de Salud.

Ferro R.; Maguiña V. (2012) Relación entre habitos alimentarios e indice de masa corporal en estudiantes de una universidad pública según área de estudio. UNMSM. Perú.

Iglesias MT, Escudero E. (2010). Evaluación nutricional en estudiantes de enfermería. Nutrición Clinica. Dietética y Hospitalaria. 30(3):21-26.

Laguado E., Gómez MP. Estilos de vida saludable en estudiantes de Enfermeria en la Universidad Cooperativa de Colombia. Hacia promoción de salud. $2014 ; 19(1): 68-83$.

Ministerio de Salud. Promoviendo universidades saludables. Dirección General de Promoción de la Salud. Dirección de Educación para la salud Lima: 2010.

Montero A., Úbeda N. (2006) Evaluación de los hábitos alimentarios de una población de estudintes universitarios en relación con sus conocimientos nutricionales. Universidad San Pablo España.

Pi Romina, Vidal Paula (2013) Estado nutricional en estudiantes universitarios: su relación con número de ingestas alimentarias diarias y el consumo de macronutrientes Facultad de Ciencias Médicas, Universidad Nacional de Córdoba, Argentina.

Sepúlveda AR, Carrobles G. (2008). Género, la escuela y las diferencias años académicos entre los estudiantes universitarios españoles con alto riesgo de desarrollar un trastorno alimentario: un estudio epidemiológico.

Torres B., Dura M. (2011). Análisis del estado antropométrica y nutricional de estudiantes de Enfermería. Universidad de Cantabria, Santander. España.

Troncoso C., Doepking C. (2011) ¿Es importante la alimentación para los estudiantes universitarios?. Facultad de Medicina, Universidad Católica de la Santísima Concepción, Concepción, Chile. 


\title{
Modelo Teórico de la Célula
}

\author{
Theoretical Model of Cell \\ Juan Carlos Broncano Torres¹, Mirtha Sussan Trejo López
}

\section{RESUMEN}

Este artículo se enmarca en el ámbito de la justificación del marco teórico que la biología requiere para comprender el carácter sistémico de sus objetos de estudio, así como, de conjeturar acerca de las posibles relaciones que existe entre el todo y las partes con la finalidad de proponer nuevas líneas de investigación.

Recordemos que a inicios del siglo XX, la Biología fue fuertemente criticada por el Círculo de Viena, Por carecer de leyes, y tener un empobrecido grado de matematización en sus sistemas de clasificación y la imposibilidad de falsar muchas de sus hipótesis. (Lorenzano, 2001; 2002).

Piaget (1979) expresó en su libro Introducción a la epistemología genética que:

"Al igual que la físico-química, la biología clasifica los objetos sobre los que trabaja, dilucida sus relaciones en forma de leyes e intenta explicar causalmente estas clasificaciones y leyes. Sólo que la estructura de estas clases en lugar de alcanzar en todos los casos y con mayor o menor facilidad un nivel matemático, conserva a menudo un carácter cualitativo, o simplemente lógico".

Inmediatamente como una respuesta a este rechazo en el año 1932 se formó en Cambridge un grupo de investigadores con el nombre de "Biotheoretical Gatherings" o también "Theoretical Biology Club", y cuyos participantes principales fueron J. H. Woodger, J. Needham, C.H. Waddington, D. M. Wrinch, J. D. Bernal. Woodger, naciendo así la Biología Teórica, como un campo que defiende la necesidad de esclarecer los fundamentos teóricos de la biología.

Desde su origen se destaca por preocuparse especialmente del problema de la organización biológica y por la elaboración de herramientas metodológicas (primero matemáticas, después también computacionales) para su tratamiento científico.

Según Hempel (1965), la explicación parte de una declaración de un fenómeno a ser explicado y encuentra un conjunto de leyes ()nLLL, .....,21 y enunciados ()nEEE,....,21 en los hechos antecedentes ()nAAA,......,21 que implican una declaración.

Lorenzano (2001), destaca que la Biología carece de unicidad en el lenguaje, lo que es relativamente frecuente para numerosas ciencias:

"En lugar de encontrarnos con un único lenguaje que nos permita expresarlas [a las ciencias biológicas], nos encontramos con una gran variedad de lenguajes específicos a ciertas subdisciplinas o teorías biológicas, lo cual no elimina la posibilidad de que éstos se relacionen de algún modo entre sí".

\section{ABSTRACT}

This article is part of the scope of the justification of the theoretical framework that biology needed to understand the systemic nature of its objects of study as well as, of guessing about possible relationships between the whole and the parts in order to propose new lines of research.

Recall that in the early twentieth century, biology was strongly criticized by the Vienna Circle Lacking laws, and have a degree of mathematization impoverished in their classification systems, and the inability to falsify many of their hypotheses. (Lorenzano, 2001; 2002).

Piaget (1979) stated in his book Introduction to genetic epistemology:

"Like physical chemistry, biology classifies objects on which it works, elucidates their relations in the form of laws and attempts to explain causally these classifications and laws. Only the structure of these classes instead of achieving in all cases and more or less ease a mathematical level, often retains a qualitative, or simply logical character."

Immediately as a response to this rejection in 1932 was formed in Cambridge a group of researchers with the name "Biotheoretical Gatherings" or also "Theoretical Biology Club" and whose main participants were J. H. Woodger, J. Needham, C.H. Waddington, D. M. Wrinch, J. D. Bernal. Woodger, and thus the Theoretical Biology, as a field that defends the need to clarify the theoretical foundations of biology.

Since its origin is known for worry especially the problem of biological organization and the development of methodological tools (first mathematics, later also computer) for scientific treatment.

According to Hempel (1965), the explanation of a statement of a phenomenon to be explained and finds a set of laws ()$n L L L, \ldots \ldots, 21$ and statements ()$n E E E, \ldots \ldots, 21$ in the background facts()nAAA,......,21 that involve a statement.

Lorenzano (2001) points out that biology lacks uniqueness in language, which is relatively common to many sciences:

"Instead of meeting a unique language that allows us to express [the biological sciences], we find a variety of specific to certain sub-disciplines or biological theories languages, which does not eliminate the possibility that they are related in some way each".

\footnotetext{
${ }^{1}$ Facultad de Ciencias. Universidad Nacional José Faustino Sánchez Carrión. Lima- Perú.
} 


\section{INTRODUCCIÓN}

Los diversos planteamientos sobre la teoría del desarrollo científico se asientan en el supuesto de la invariancia del significado de los términos científicos. Por ejemplo para C. Popper el carácter teórico de todos los enunciados científicos ha llevado a abandonar la distinción tradicional absoluta entre términos teóricos y términos observacionales, sustituyéndola por una distinción, contextualizada en relación con una teoría dada, entre aquellos términos que funcionan como teóricos para la teoría en cuestión y aquellos que se consideran no teóricos en relación con la misma teoría (lo que no implica que no puedan ser teóricos en una teoría previa o diferente). Por lo que se refiere, al problema del contenido empírico o factual de las teorías científicas.

Por otro lado R. Carnap, propone una nueva concepción del pensamiento analítico de la ciencia donde se considera, reconstruir la estructura lógica de las teorías científicas de acuerdo con el ideal de la unidad de la ciencia. Sus componentes básicos son el método de reconstrucción axiomática de las teorías científicas en el marco del lenguaje formal de la lógica (predominantemente de la lógica clásica), la distinción en los lenguajes de la teoría empírica entre términos (y enunciados) teóricos y términos (y enunciados) observacionales, y finalmente el conjunto de tesis epistemológicas (fenomenalismo, empirismo, etc.) con las que se intenta resolver el problema de las relaciones entre el lenguaje de las teorías y la realidad a la que se refieren.

Por el contrario para P. Suppes se debe sustituir la axiomatización de una teoría mediante un sistema formal, al que luego se le busca una interpretación adecuada, por la definición semiformalizada de un predicado conjuntista. La idea fundamental de este nuevo enfoque de la filosofía de la ciencia se puede enunciar así: " una teoría científica consta de dos componentes principales, un núcleo $K$ y conjunto $/$ de aplicaciones propuestas de la teoría. $K$ es una estructura matemática; I es el conjunto de sistemas que constituyen modelos de".

Por tanto al analizar una teoría científica es poner de manifiesto su estructura matemática o núcleo estructural, así como localizar el conjunto de sus aplicaciones. De esta manera se combinan en la teoría de la ciencia el análisis formal y la tarea histórico-pragmática de localizar las aplicaciones paradigmáticas que constituyen un componente esencial de la teoría.

La Biología como ciencia de la vida queda enmarcada bajo esta línea de pensamiento, por tanto ella debe tener una teoría general de la vida comparable a la teoría física de los campos electromagnéticos o la teoría cuántica de las uniones químicas.

La investigación propuesta en este artículo se centra en dos aspectos fundamentales, el desarrollo de un cálculo lógico y el intento de axiomatización de cierto sector de la biología como la Citología en general y la célula en particular, así como el carácter formal de sus implicaciones esclarecedoras en el seno de la ciencia proponiendo la posibilidad de desarrollar una teorización y un tratamiento de aquellas estructuras y funciones de los seres vivos que son logizables y algeibrizables.

\section{LACÈLULA}

Según el diccionario de la RAE (Del lat. cellula, dim. de cella, hueco). f. Biol. Es la unidad fundamental de los organismos vivos, generalmente de tamaño microscópico, capaz de reproducción independiente y formada por un citoplasma y un núcleo rodeados por una membrana.

En textos especializados de Citología (Murray, 1993) y (Brown y Rothery, 1993), la célula es el vehículo a través del cual se transmite la información hereditaria que define cada especie y que además contiene la maquinaria necesaria para obtener materiales del ambiente y generar una nueva célula a su imagen, que contendrá una nueva copia de la información hereditaria.

\section{MODELO TEÓRICO DE LACÉLULA \\ A. Modelo Matemático.}

Un modelo matemático es un objeto, concepto o conjunto de relaciones, que se utiliza para representar y estudiar de forma simple y comprensible una porción de la realidad empírica.

El primer modelo matemático que se conoce data del año 1190, éste fue propuesto por Leonardo de Pisa, quien realizó demostraciones retóricas y usó segmentos de recta como representación de cantidades, a partir de entonces su alcance se ha incrementado, afinando sus usos y aplicaciones, como en la dinámica de poblaciones y disciplinas como la biología hasta llegar a la epidemiología.

\section{B. Estructura algebraica de un sistema vivo}

El libro de SNEED, The Logical Structure of Mathematical Phisics, y el de W. STEGMÜUJER, The Structure and Dynamics of Theories, son hitos importantes en la axiomatización conjuntista de las teorías empíricas, pues proporciona un método de análisis, con la intención de dar cuenta de la estructura y la dinámica de las mismas.

En el campo de las ciencias biológicas, ha sido la teoría genética la que ha utilizado este tipo de formalizaciones, como por el ejemplo el desarrollado por J.H. Woodger, en la axiomatización enunciativa de la genética mendeliana y el de W. Balzer en la axiomatización molecular.

Desarrollando así un intento de teorización y cualitativo de aquellas estructuras y funciones de los seres estableciéndose de esta manera una nueva metodología de trabajo en la biología.

La estructura interna del razonamiento biológico no está al margen como tal, y por lo tanto pensar en 
la necesidad de formación en los principios y procedimientos deductivos de la lógica no debe estar al margen de las pretensiones formativas de un biólogo.

\section{- Orgánulo}

En Biología celular es una estructura elemental, en esta axiomatización es una entidad abstracta que no consta de componentes y que es susceptible de tomar solamente dos valores que se excluyen, como son todo o nada, verdad o falsedad, sí o no, abierto o cerrado, par o impar, etc., al cual la denotaremos como: Orgcellx

\section{- Organelas celulares}

Es el conjunto de todos los orgánulos que tienen diversas especializaciones y con morfología variada. Aquí se considera como una cantidad booleana general. al cual la denotaremos como:

$$
\text { Orgcell }=\bigcup_{i \in I_{m}} \text { Orgcell }_{i}, \operatorname{con}_{m} \mathrm{C} N
$$

\section{- Citoplasma}

En biología consiste en una dispersión coloidal muy fina de aspecto granuloso cuya función es albergar los orgánulos celulares y contribuir al movimiento de estos. En esta axiomatización se considera como un sistema enlace que denotaremos como: $\mathrm{C}=$ (Orgcell, $\bar{R}$ ) en el que $\bar{R}=\left\{R_{1}, R_{2}\right\}$, siendo:

\section{$\mathrm{R}_{1}$ : Función especifica de Orgacellx.}

La función especifica de Orgcellx es el conjunto de aquellas actividades de Orgcellx que no es capaz de realizar ningún Orgacellx en $\mathrm{C}$.

\section{$\mathrm{R}_{2}$ : Proyecto teleonómico de Orgcellx.}

El proyecto teleonómico es todo aquello que contribuya al éxito de la función especifica de al éxito de la función especifica de Orgcellx.

Ademas $\bar{R}$ satisface dos axiomas:

\section{Axioma 01}

Dado Orgcellx $\bar{R}$ Orgcellz entonces Orgcellx = Orgcellz.

\section{Axioma 02}

Dado Orgcellx C, entonces existe Orgcellz $\quad$ tal que no se relacionan bajo $\bar{R}$.

\section{- Célula}

La célula se representa como la tripleta: Cell $=(C, M, R)$ en el que: $\mathrm{C}=$ (Orgcell, $\overline{\mathrm{R}})$.

M: Es el conjunto de cosas concretas, distintas de $\mathrm{C}$ que actuán sobre ella, o son afectadas por ella.
$R$ : Es el conjunto de relaciones entre los elementos de $C$ así como entre éstos y componentes de $M$, dicho conjunto incluye por lo menos una conexión.

\section{Proposición 01}

Dado el conjunto numérico $I_{n}=\{p, n<n\}$ y el conjunto de todas las células Cell, entonces la función: $\lambda: I_{n} \rightarrow$ Cell

$$
p \rightarrow \lambda(p)=\text { Cell }_{p}
$$

Es biyectiva

\section{Comunicación Celular}

Según Morgan (1989), las células de los organismos pluricelulares están provistas de mecanismos de señalamiento que les permiten recibir, procesar y responder a diferentes estímulos.

Estos mecanismos aseguran que cada célula funcione y responda a una gran diversidad de estímulos y lo haga coordinadamente con las otras células del organismo, manteniendo al organismo como entidad unitaria, a pesar de que posee millones de células distintas. Dicha coordinación sólo puede lograrse mediante una compleja red de comunicación celular.

\section{-Alfabeto Celular.}

Por la razón expuesta anteriormente definiremos el alfabeto celular como el conjunto $\Sigma=\{0,1\}$

\section{- Palabras a nivel Celular.}

Es una secuencia finita de ceros y unos seleccionados de $\Sigma$ y forman un conjunto $\Sigma^{*}$ tal que $\Sigma^{*} \quad \Sigma$.

\section{- Palabra celular vacía}

Desde que los genes están sometidos procesos de mutación y otros procesos de reorganización que provocan un cambio en la expresión fenotípica de éstos, entonces admitimos la existencia de una única cadena que no tiene símbolos, la cual denominaremos cadena vacía y será denotado por 0 .

\section{Proposición 02}

Dado el conjunto de los números naturales $\mathrm{N}$ y el conjunto de todas las palabras $\Sigma^{*}$, entonces la función:

$$
\begin{aligned}
\varphi: N & \rightarrow \Sigma^{*} \\
n & \rightarrow \varphi(n)=u_{n}
\end{aligned}
$$

\section{Es biyectiva}

\section{- Longitud de una palabra}

La longitud de una cadena $u \quad \Sigma^{*}$ se denota $\|\mathcal{u}\|$ y se define como el número de símbolos de $u$ (contando los símbolos repetidos). Es decir: 
$\|u\|=n \quad u=a_{1} * a_{2}{ }^{*} \ldots . . * a_{n} \quad a_{n} \quad \Sigma$

\section{- Concatenación de palabras}

Dado dos cadenas $u, v \quad \Sigma^{*}$, la concatenación de $u$ y $v$ se denota como $u * v$

\section{Proposición 03}

La concatenación de cadenas es una operación asociativa. Es decir $u, v, w \quad \Sigma$ * entonces $(u * v) *$ $w=u *(v * w)$.

- Lenguaje Celular L sobre el alfabeto $\Sigma$ es un subconjunto de $\Sigma^{*}$ y está definido sobre los genes en código binario contenido en los cromosomas de cada Cell

\section{- Información Celular.}

Un Modelo de Información celular (se supone la existencia de una célula emisora y receptora) es una cuádrupla $D, Q, F, R\left(q_{i}, d_{j}\right)$ donde:

- $D$ es un conjunto formado por la representación de la comunicación en la célula receptora.

- $Q$ es un conjunto formado por consultas, es decir la representación de la información que la célula emisora necesita.

- F es un marco o modelo de representación de la información, las consultas, y las relaciones existentes entre ellos.

- R $\left(q_{i}, d_{j}\right)$ es una función (ranking) que asocia un número real a cada consulta $q_{i}$ de $D$.

\section{- Posición celular}

Cell $I_{p}$ es un sistema con capacidad tanto de detección de las propiedades de su entorno, como de movimiento deberá evaluar, decidir y ejecutar los procesos necesarios con el fin de llevar a cabo su función específica.

Denotaremos por $\mathrm{x}(\mathrm{t})$ a la posición de la célula Cell $X$ en instante $t$.

\section{- Tiempo de vida celular.}

Las células de un organismo no viven indefinidamente y su vida media depende del tipo celular.

El número de células que componen un tejido en un organismo adulto permanece, dentro de ciertos límites, constante.

Algunos tipos de muerte celular conllevan la activación de mecanismos específicos que dictan que se produzca un suicidio o muerte celular programada.

Estos mecanismos fisiológicos de muerte son empleados por los organismos multicelulares durante el desarrollo, la morfogénesis y en el mantenimiento de la homeostasis tisular en el organismo adulto, así como para controlar el número de células y eliminar células infectadas, mutadas 0 dañadas. Por tanto el tiempo de existencia de $\mathrm{Ce}_{p} \mathrm{X}$ esta dado por el intervalo

$t^{p} e(x)=\left[t_{0}, t_{f}\right]$

\section{- Estado celular.}

Se entenderá por estados de una célula en un instante $t$, al conjunto de todos los genes que la célula tiene en ese instante. Denotaremos por

$x_{e}(t)=u$, donde $u=a_{1} * a_{2} * \ldots * a_{n} ; \quad a_{n} \quad \Sigma$, con

$t \quad t^{p} e(x)$. Al estado en el que se encuentra Cell en el instante $t$.

\section{Proposición 04}

Sea el conjunto $M=\left\{C e l l_{p} x /\left\|x^{p} e(t)\right\|=n\right\}$, entonces $d: M x M \quad Z^{*}$

$$
d\left(x^{p} e(t), y^{p} e(t)\right)=\Sigma\left(1-\delta_{\mathrm{x} \mathrm{e(t)y} \mathrm{e}(\mathrm{t})}(\mathrm{i})=\left\{\begin{array}{l}
1, \text { si } a_{i}=b_{j} \\
0, \text { en otros casos }
\end{array}\right.\right.
$$

у $x_{e}(t)=a_{1} * a_{2} * \ldots * a_{n} ; y_{e}(t)=b_{1} * b_{2} * \ldots . . * b_{n}$ es una métrica celular.

\section{Observación}

$d\left(x^{p} e(t) ; y^{p} e(t)\right)$ representa el número de genes de Cell $_{x p}$ no comunes a los genes de Celly respectivamente, luego cuando más pequeño es el valor de $d\left(x^{p} e(t) ; y^{p} e(t)\right)$, más grande es la coincidencia entre las cadenas genéticas de las células $\mathrm{Cell}_{p} x \quad y \mathrm{Cell}_{q} y$.

\section{CONCLUSIÓN}

El tema tratado en este artículo, es de gran valor para la enseñanza de las ciencias Biológicas. Por su efecto en la reforma de las estructuras conceptuales, así como en la concepción de la actividad investigativa, puesto que, el aspecto lingüístico, inherente a ella, se traduce en un conjunto de proposiciones sobre un ámbito concreto de objetos.

Distinguiéndose así dos tipos de objetos unos reales los cuales se nos es accesible gracias a la acción investigativa y otros ideales entendidos como constructos ideales (puramente mentales) que vienen a existir sólo como resultado de la actividad mental enmarcados en el contexto de la lógica y las matemáticas.

Así la actividad de la enseñanza-aprendizaje de las ciencias Biológicas permitirá formular algunas hipótesis fundadas en la estructura lógica matemática, cuya convalidación puede dar lugar a futuros trabajos de investigación. 


\section{REFERENCIAS BIBLIOGRÁFICAS}

Alberts, B.; Johnson, A.; Lewis, J.; Roberts, K. y Walter, P. (2010). Biología Molecular de la Célula. Omega Quinta Edición.

Attwood, T.K. y Parry-Smith, D.J. (2002). Introducción a la Bioinformática. México: Prentice Hall.

Boudon, R. (1992). Para qué sirve la noción de estructuras. Aguilar: Madrid.

Boudon, R. (1994). L'analyse mathematique des faits sociaux. París: Plon.

Brown, D. y Rothery P. (1993). Models in Biology: mathematics, statistics and computing. Chicheste: John Wiley \& Sons.

Bunge, M. (1991). General systems and holism. Dordrecht y Boston: D. Reidel Publ. Co.

Hempel, C. (1965). La explicación científica. Buenos Aires: Paidós.

Lorenzano, P. (2001). Sobre la unidad de las ciencias biológicas. Signos Filosóficos 5 (enero-junio) 121131.

Lorenzano, P. (2002). Presentación de la concepción científica del mundo: el Círculo de Viena. REDES, 9(18) 103-149.

Lwoff, B. (1994). Biological order. MIT, Cambridge,

Mass.

Lloret, M. (1996). Sistemas vivos y sus modelos matemáticos, Modelización de un ecosistema. Tesis Doctoral. Universidad de Alicante. España.

Morgan, N. G. (1989). Cell signaling. Milton Keynes, UK. Open University Press, p 1-30.

Murray, J. D. (1993). Mathematical biology. Berlín, Springer.

Piaget, J. (1979). Introducción a la epistemología genética. Buenos Aires: Paidós.

Piaget, J. (1993). El estructuralismo. Buenos Aires. Argentina: Proteo. 
BIG BANG FAUSTINIANO es una de las revistas del Vicerrectorado de Investigación de la Universidad Nacional José Faustino Sánchez Carrión, tiene por finalidad difundir el conocimiento científico multidisciplinario, entre los profesores y alumnos de la Universidad y de otras Universidades.

\section{PROCESO EDITORIAL}

La revista acepta artículos enviados para su publicación. Estos serán revisados por el comite editorial. La revisión tiene el objetivo de asegurar que los artículos se adaptan a las normas y políticas de la revista. En caso contrario, serán devueltos al autor para su corrección quien deberán reenviar el trabajo en un plazo no superior a 3 días debidamente corregidos. Después que el articulo sea aprobado por el comite editorial, sera enviado a evaluación externa. Al finalizar esta fase, el comite editorial tomara una decisión sobre su publicación.

\section{PRINCIPIOS ÉTICOS EN LA INVESTIGACIÓN Y PUBLICACIÓN}

La revista controlara cualquiera de las siguientes practicas deshonestas:

- Fabricación de resultados (cuando se presentan datos ficticios).

- Falsificación (cuando los datos son manipulados o cambiados).

- Omisión (cuando se oculta información deliberadamente).

- Plagio (cuando se presenta información, ideas o datos de autores como propios)

Se tendrá en cuneta dos formas de plagio: la copia directa de un texto sin uso de comillas ni citación de la fuente; la modificación de algunas palabras del texto, parafraseando o sin suficiente identificación de la fuente.

lallazgos serán notificados a los autores para la subsanación

\section{REQUISITOS DE PRESENTACIÓN}

- El autor presentara una solicitud dirigida al Vicerrector de Investigación, con los recibos respectivos, donde indicara que autoriza la publicación de su articulo en la Revista de Big Bang Faustiniano. Ademas deberá adjuntar un ejemplar impreso y en un $C D$, teniendo en cuenta las características establecidas a lo largo del documento.

- Pueden participar hasta 4 integrantes por articulo y deben aplicar las Normas A.P.A. (sexta edición). El autor deberá respetar el cierre de la edición, y presentar su articulo en el margen de tiempo establecido.

- Se presentara un máximo de texto diez paginas (no diez hojas) tamaño A4, interlineado 1,5 con márgenes de 2,5 cm. El formato es letra Arial, tamaño 10.

os artículos científicos se presentaran adjuntando una constancia de haber sido revisado en su contexto gramatical por un filólogo, lingüista, literario y/o comunicador social.

\section{ESTRUCTURADELARTICULO}

Se encuentra estructurado de la siguiente forma:

\section{Titulo en español (y traducción del mismo al ingles)}

- Debe ser conciso y especifico, así mismo, no debe exceder las 15 palabras.

- El titular de los artículos estará realizado con cualquiera de las variaciones de tipográfica de Arial.

- No partir las palabras en un titulo.

- No emplear el punto final en un titulo.

- El titulo no debe ser la primera frase del texto.

- El titulo debe representar toda la temática del articulo presentado.

\section{Autores (tesistas y asesores)}

- Si son varios autores, se debe colocar los nombres y apellidos completos en orden de contribución del trabajo. Cada autor sera identificado con un superíndice (si son de la misma institución y facultad se empleara el mismo número, caso contrario serán número distintos).

- Al terminar el articulo, el autor escribirá su nombre completo, grados académicos, cargo (si lo tuviera), especialidad y facultad a la que pertenece. De ser externo a la Universidad, deberá indicar la institución, cargo y país al que pertenece.

- Al finalizar el articulo, deberá consignar sus datos personales como DNI, correo electrónico, Nro. de celular, teléfono fijo, dirección de domicilio.

3. Resumen: Debe estar estructurado en un solo párrafo, teniendo en cuenta el siguiente orden: objetivo, método, resultados y conclusiones. La redacción no debe contener, formular, tablas, resaltar que la redacción debe estar en tiempo pasado. Debe tener 250 palabras como máximo, además contener objetivos, métodos, resultados y conclusiones, en forma estructurada e incluir las palabras clave.

Palabras claves: Debe contar con un mínimo de 3 y un máximo de 6 palabras simples y/o compuestas.

4. Abstract: En la traducción del articulo al idioma inglés.

Keywords: Palabras clave traducidas al ingles.

5. Introducción: Racionalidad del tema de investigación, justificación, antecedentes, objetivos.

6. Materiales y métodos: Procedimientos utilizados, incluyendo forma de análisis de datos.

7. Resultados: Las tablas y figuras se utilizaran solo cuando contribuyan a la nitidez de la explicación.

8. Discusión: Se plantea la interpretación y justificación de los resultados y los contrasta con trabajos encontrados en la revisión bibliográficas y con la hipótesis.

\section{Agradecimientos}

10.Referencias bibliográficas(Norma A.P.A. Sexta Edición).

- Notas importantes

\section{- Imágenes}

- Las fotografías presentadas deben estar en formato JPG y adjuntar en un archivo aparte del formato word (no incluir la imagen dentro del texto en word), siempre considerar el pie de foto. Deben ser originales y autenticadas.

\section{- Tablas y figuras}

- Deben especificar el nombre de la tabla o figura, asi como también la fuente de consultar (Es válido: Elaborado por el autor)

\section{ÉTICADEL INVESTIGADOR}

a) Las implicancias a favor o en contra, están relacionadas al autor.

La Revista no se solidariza necesariamente con los puntos de vista del autor.

b) Cuando los artículos no se encuadren en el mensaje de valores y fines institucionales, no serán formados en cuenta.

c) Los autores presentarán una declaración jurada (con firma y huella digital) sobre la originalidad de la investigación propuesta, asumiendo sus responsabilidad respectivas.

d) El Comité de Ética realizara acciones preventivas y de supervisión en forma permanente.

\section{OTROS TIPOS DE ARTÍCULOS}

- Original Breve

- Monografía

- Reporte de casos

- Artículos de revisión

- Carta al editor 

